\title{
Kinetics of Coalescence, Annihilation, and the q-State Potts Model in One Dimension
}

\author{
Thomas Masser and Daniel ben-Avraham* \\ Physics Department, and Clarkson Institute for Statistical Physics (CISP), \\ Clarkson University, Potsdam, NY 13699-5820
}

\begin{abstract}
The kinetics of the q-state Potts model in the zero temperature limit in one dimension is analyzed exactly through a generalization of the method of empty intervals, previously used for the analysis of diffusion-limited coalescence, $A+A \rightarrow A$. In this new approach, the q-state Potts model, coalescence, and annihilation $(A+A \rightarrow 0)$ all satisfy the same diffusion equation, and differ only in the imposed initial condition.
\end{abstract}

82.20.Mj, 05.50.+q, 05.70.Ln, 68.10.Jy

The kinetics of the $q$-state Potts model has drawn much recent attention, in particular regarding its persistence probabilities [1-10]. An understanding has emerged that, as far as persistence is concerned, the kinetics of the $q$-state Potts model in the zero temperature limit, with Glauber dynamics and in one dimension, is equivalent to that of the Ising model, when a fraction $1 / q$ of the spins are initially up [6]. It is also understood that if one regards the boundaries between domains as particles (kinks), then $q=2$ (the Ising model) corresponds to the diffusion-limited annihilation process $A+A \rightarrow 0$, while the limit $q \rightarrow \infty$ corresponds to coalescence, $A+A \rightarrow A$. Here we extend the formalism of empty intervals, previously used for the exact analysis of $A+A \rightarrow A$ [11], to annihilation, by considering the probability that an interval contains an even number of particles, and we apply this new approach also to the $q$-state Potts model. This results in one simple diffusion equation describing all three models (annihilation, Potts model, and coalescence): they differ only in the initial conditions they impose.

Consider diffusion-limited annihilation, $A+A \rightarrow 0$, in a one-dimensional lattice of lattice spacing $a$. The particles hop randomly to the nearest site to their right or left, at equal rate $\Gamma$, and annihilate immediately upon encounter. Let $G_{n}(t)$ be the probability that an arbitrary segment of $n$ consecutive sites contains an even number of particles, at time $t$. (We assume that the system is infinite and homogeneous.) A site can be either empty or occupied by a single particle, so the particle concentration is

$$
c(t)=\frac{1-G_{1}(t)}{a} .
$$

Because the reaction $A+A \rightarrow 0$ conserves the number of particles modulo 2 , the only way that the parity within an $n$-segment is changed is when particles at the edge of the segment hop out, or when particles outside of the segment hop in. To describe these events, we require $F_{n}(t)$ - the probability that an $n$-segment containing an even number of particles is followed by the presence of a particle at the $(n+1)$-th site. This can be expressed in terms of the $G_{n}$ (Fig. 1):

$$
2 F_{n}(t)=\left(1-G_{1}\right)+\left(G_{n}-G_{n+1}\right) .
$$

Likewise, $H_{n}(t)$ - the probability that an $n$-segment containing an odd number of particles is followed by a particle at the $(n+1)$-th site - is

$$
\begin{gathered}
2 H_{n}(t)=\left(1-G_{1}\right)-\left(G_{n}-G_{n+1}\right) . \\
\left.\frac{\mathrm{G}_{\mathrm{n}+1}\{}{2 \mathrm{~F}_{\mathrm{n}}+\mathrm{G}_{\mathrm{n}+1}=\mathrm{C}_{1}} \cdot\right\} \cdots \cdots \cdot \mathrm{G}_{1}+\mathrm{G}_{\mathrm{n}}
\end{gathered}
$$

Fig. 1: Computation of $F_{n}(t)$. Empty (solid) rectangles symbolize $n$-segments with an even (odd) number of particles. Empty (solid) circles represent empty (occupied) sites. The four events listed may be grouped in two different ways, yielding eq. (2a). One can also see that the probabilities of the first two events add up to $F_{n}+H_{n}=1-G_{1}$, yielding eq. (2b).

The evolution equation for $G_{n}$ is now readily obtained:

$$
\frac{\partial}{\partial t} G_{n}(t)=2 \Gamma\left(F_{n-1}-H_{n-1}+H_{n}-F_{n}\right),
$$

where the first term on the r.h.s expresses the event that a particle at site $n$ jumps out of the segment, leaving an even number of particles in the remaining $n-1$ sites (and hence in the $n$-segment); the second term denotes the same case, but when there are initially an even number of particles in the $n$-segment (that is, an odd number in the $(n-1)$-segment); and the last two terms pertain to a particle just outside of the $n$-segment, at site $n+1$, jumping in. The factor of 2 accounts for the fact that each event may occur at the left or right edge of the

*e-mail: benavraham@clarkson.edu 
segment, with equal probability. Using eqs. (2), this becomes

$$
\frac{\partial}{\partial t} G_{n}(t)=2 \Gamma\left(G_{n-1}-2 G_{n}+G_{n+1}\right) .
$$

The case of $n=1$ requires a special equation, since $G_{0}$ is undefined. Taking into account all the ways $G_{1}$ might change, one finds

$$
\frac{\partial}{\partial t} G_{1}(t)=2 \Gamma\left(1-2 G_{1}+G_{2}\right) .
$$

Thus, eq. (3) may be understood to be valid for all $n \geq 1$, provided that one uses the boundary condition

$$
G_{0}(t)=1 .
$$

Additionally, since the $G_{n}$ are probabilities, we have

$$
0 \leq G_{n}(t) \leq 1 .
$$

We now turn our attention to the $q$-state Potts model in one dimension, at the zero temperature limit and with Glauber dynamics. (This is also known as the voters model, with $q$ opinions.) Each site can assume one of $q$ states, $s_{1}, s_{2}, \ldots, s_{q}$. The model possesses $q$ equivalent ground states, where all the sites are in the same state. If the system is started away from equilibrium, then neighboring sites of differing states react according to the scheme:

$$
s_{i} s_{j} \rightarrow \begin{cases}s_{i} s_{i} & \text { rate } \Gamma \\ s_{j} s_{j} & \text { rate } \Gamma\end{cases}
$$

Hence the interface between domains of different states may be regarded as particles (kinks) that perform regular random walks. Let the kinks between a domain of state $s_{i}$ on the left, and a domain of state $s_{j}$ on the right, be denoted by $A_{i j}$. The dynamics (5) implies the following immediate reactions between colliding kinks:

$$
\begin{gathered}
A_{i j}+A_{j k} \rightarrow A_{i k}, \\
A_{i j}+A_{j i} \rightarrow 0 .
\end{gathered}
$$

Notice that the inner indices ( $j$, in the cases above) must be the same for kinks to meet.

Focusing now on the kinks, and assuming an infinite homogeneous system, let $G_{n}(t)$ be the probability that an arbitrary $n$-segment contains kinks such that their total number of indices of each of the q kinds is even, at time $t$. Because the reactions (6) conserve the parity of the numbers of indices, reactions within an $n$-segment do not affect $G_{n}$. Thus, $G_{n}$ changes only through kinks that hop into or out of the segment, exactly as in the case of the annihilation process. (Note that a kink must have different indices, so the parity is always affected by such transitions.) It follows that the $G_{n}$ obey eq. (3), with the boundary conditions (4). Finally, if one merely counts the kinks, ignoring their indices, then their concentration is given once again by eq. (1).

For $q=2$ the process (6a) never takes place (it requires at least three states) and the problem reduces to that of the annihilation process, $A+A \rightarrow 0$. On the other hand, the probability that the sites to the left and right of the two kinks are in the same state $s_{i}$ tends to zero as $q \rightarrow \infty$. In that case process (6b) never takes place and the model is then analogous to coalescence, $A+A \rightarrow A$. For $2<q<\infty$ kinks may either coalesce or annihilate, and we have a mixture of the two processes. Annihilation, coalescence, and the $q$-state Potts model are all described by the same (discrete) diffusion equation, eq. (3), and the same boundary conditions, eqs. (4).

The difference between the three models is in their implied initial condition. Assume, for example, initial conditions where each site is randomly in one of its $q$ possible states. The probability that an $n$-segment contains even numbers of indices equals the probability that the first and last site in the segment are in the same state. For the case of initially random states this yields

$$
G_{n}(0)=\frac{1}{q}
$$

While the initial state above is natural, it is by no means unique. Consider, for example, the initial state of Fig. 2a, for which

$G_{n}(0)= \begin{cases}\frac{m-n}{m} & 0<n \leq m, \\ 1 & k q m<n \leq(k q+1) m, \quad k=1,2, \ldots \\ 0 & \text { otherwise. }\end{cases}$

This corresponds to kinks that are initially evenly spaced, $m$ sites apart from each other. Notice, however, that the initial states in between the kinks could be chosen differently (Fig. 2b), thus dictating a different form of $G_{n}(0)$ and different kinetics!

\begin{tabular}{l|l|l|l|l|l|l}
\hline 3 & 1 & 2 & 3 & 1 & 2 & 3 \\
(a) & \\
\hline 1 & 3 & 2 & 3 & 2 & 1 & 3
\end{tabular}

(b)

Fig. 2: Possible initial configurations for the $q$-state Potts model. Here the system consists of alternating domains of equal length $m$, which corresponds to equally spaced kinks (the vertical bars), $m$ sites apart from each other. In (a) the states of the domains alternate periodically: $1,2, \ldots, q, 1,2, \ldots$ while in (b) the states are random (but do not repeat). In this illustration, $q=3$.

Eq. (3) may be solved by standard techniques, for example, by Laplace-transforming with respect to time, fitting an exponential solution to the resulting difference equation, and finally inverting the Laplace-transformed 
solution [12]. With the boundary conditions (4) and the natural initial condition (7), one obtains:

$$
G_{n}(\tau)=\frac{1}{q}+\frac{q-1}{q} \int_{0}^{\tau} n e^{-2 \tau^{\prime}} I_{n}\left(2 \tau^{\prime}\right) \frac{d \tau^{\prime}}{2 \tau^{\prime}},
$$

where $\tau=2 \Gamma t$ and $I_{n}(\cdot)$ is the modified Bessel function of order $n$ [13]. In particular, the probability that a site contains a kink, is

$$
1-G_{1}(\tau)=\frac{q-1}{q}\left(1-\int_{0}^{\tau} e^{-2 \tau^{\prime}} I_{1}\left(2 \tau^{\prime}\right) \frac{d \tau^{\prime}}{2 \tau^{\prime}}\right) .
$$

Thus, with natural initial conditions, the density of kinks in the $q$-state Potts model has a simple dependence on $q$ : it is exactly equal to the density of particles in the coalescence model, $A+A \rightarrow A$, times $(q-1) / q$.

For completeness, we note that for low initial concentrations of kinks, passage to the continuum limit is justified. Putting $x=n a, G_{n}(t) \rightarrow G(x, t)$, and $\Gamma=D / a^{2}$ in eq. (3), and taking the limit $a \rightarrow 0$, one obtains the diffusion equation

$$
\frac{\partial}{\partial t} G(x, t)=2 D \frac{\partial^{2}}{\partial x^{2}} G(x, t) .
$$

The boundary condition (4a) becomes $G(0, t)=1$, and the concentration is obtained from the continuum limit of eq. (1); $c(t)=-\partial G /\left.\partial x\right|_{x=0}$. Consider, for example, an initial concentration $c_{0}$ of randomly placed kinks, where the states of the domains in between are random (but do not repeat). Following simple combinatorial arguments, it can be shown that this corresponds to the initial condition

$$
G(x, 0)=\frac{1}{q}+\frac{q-1}{q} e^{-\frac{q}{q-1} c_{0} x} .
$$

Solving for the concentration, we obtain

$$
c(t)=c_{0} e^{z^{2}} \operatorname{erfc}(z), \quad z=\frac{q}{q-1} c_{0} \sqrt{2 D t},
$$

which has the expected long-time asymptotic behavior

$$
c(t) \sim \frac{q-1}{q} \frac{1}{\sqrt{2 \pi D t}}, \quad t \rightarrow \infty .
$$

We have introduced a new method for the analysis of dynamic lattice models in one dimension. This approach brings to light the connection between diffusion-limited coalescence, $A+A \rightarrow A$, diffusion-limited annihilation, $A+A \rightarrow 0$, and the $q$-state Potts model with Glauber dynamics in the zero-temperature limit. All three models are described by the same diffusion equation; the reaction between particles (or kinks) is manifested in the boundary condition (4a), again, shared by all three models; and the only differences arise from the different initial conditions implied in each case.

For random initial conditions we obtain results which are in agreement with what is known from studies of persistence, but the new formalism can also handle correlated initial conditions, where the three models might differ in nontrivial ways. Moreover, the new approach can be extended to inhomogeneous situations, along the same lines as the method of empty intervals (IPDF) [14].

We thank L. Glasser and D. Kessler for edifying discussions, and we gratefully acknowledge NSF support of this work (PHY-9820569).

[1] B. Derrida, A. J. Bray, and C. Godrèche, "Nontrivial exponents in the zero-temperature dynamics of the 1D Ising and Potts models", J. Phys. A 27, L357 (1994).

[2] D. Stauffer, "Ising spinodal decomposition at $T=0$ in one to five dimensions", J. Phys. A 27, 5029 (1994).

[3] B. Derrida, "Exponents appearing in the zero-temperature dynamics of the 1D Potts model", J. Phys. A 28, 1481 (1995).

[4] B. Derrida, V. Hakim, and V. Pasquier, "Exact firstpassage exponents of $1 \mathrm{D}$ domain growth - relation to a reaction-diffusion model", Phys. Rev. Lett. 75, 751 (1995).

[5] B. Derrida, V. Hakim, and V. Pasquier, "Exact exponent for the number of persistent spins in the zero-temperature dynamics of the one-dimensional Potts model", J. Stat. Phys. 85, 763 (1996).

[6] E. Ben-Naim, L. Frachebourg, and P. L. Krapivsky, "Coarsening and persistence in the voter model", Phys. Rev. E 53, 3078 (1996).

[7] D. H. Zanette, "Distribution of persistent sites in diffusing systems", Phys. Rev. E 55, 2462 (1997).

[8] G. Manoj and P. Ray, "Dynamic scaling in the spatial distribution of persistent sites", preprint, cond-mat/9901130 (1999); "Spatial distribution of persistent sites", preprint, cond-mat/0003203 (2000); "Scaling and fractal formation in persistence", J. Phys. A 33, L109 (2000).

[9] S. Jain and H. Flynn, "Scaling and persistence in the two-dimensional Ising model", preprint, cond-mat/0004148 (2000).

[10] A. J. Bray and S. J. O'Donoghue, "Unusual dynamical scaling in the spatial distribution of persistent sites in $1 \mathrm{D}$ Potts models", preprint, cond-mat/0005180 (2000).

[11] M. A. Burschka, C. R. Doering, and D. ben-Avraham, "Transition in the relaxation dynamics of a reversible diffusion-limited reaction", Phys. Rev. Lett. 63, 700 (1989); D. ben-Avraham, "The method of interparticle distribution functions for diffusion-reaction systems in one dimension", Mod. Phys. Lett. B 9, 895-919 (1995); "The coalescence process, $A+A \rightarrow A$, and the method of interparticle distribution functions", in Nonequilibrium Statistical Mechanics in One Dimension, V. Privman, ed., pp 29-50 (Cambridge University Press, 1997); D. ben-Avraham and S. Havlin, Diffusion and Reactions in Fractals and Disordered Systems, in press (Cambridge University Press, 2000).

[12] E. Abad, H. L. Frisch, and G. Nicolis, "1D lattice dynamics of the diffusion limited reaction $A+A \rightarrow A+S$ : transient behavior", preprint, cond-mat/0002432 (2000). 
[13] M. Abramowitz and I. M. Stegun, eds., Handbook of Mathematical Functions, (Dover Publications, New York, 1964).
[14] C. R. Doering, "Microscopic spatial correlations induced by external noise in a reaction-diffusion system", Physica A 188, 386 (1992). 